\title{
Crystal-Morphological Identification of Varicose Disease Stages of Lower Limbs
}

\author{
Larisa A. Kurbatova ${ }^{1, *}$, Iuriy M.Smirnov ${ }^{2}$, Margarita B. Petrova ${ }^{1}$, Elena A. Kharitonova ${ }^{1}$, Natalia V. Pavlova ${ }^{1}$, \\ Nadezhda V. Ilyashenko ${ }^{1}$ \\ ${ }^{1}$ Department of Biology, Tver State Medical Academy, Tver, Russia \\ ${ }^{2}$ Department of Crystallization, Tver State University, Tver, Russia \\ *Corresponding author: biologiatgma@mail.ru
}

Received December 30, 2012; Revised February 21, 2013; Accepted March 10, 2013

\begin{abstract}
In work method for differentiation of varicose veins of lower extremities stages was used. This method is founded on comparative analysis of traditional histological and crystal-morphological methods. Dependency crystal-morphological drawing of the textures veins of lower extremities from stage varicose disease is observed.
\end{abstract}

Keywords: crystal-morphological method, varicose veins, crystallized areas of vein

\section{Introduction}

At the present-day stage, medicine increasingly involves the achievements of physic-mathematical sciences into the sphere of diagnostics, treatment and disease prophylaxis. In this connection crystalmorphological diagnostics method that is based on the concentration and objects components change reflecting on the habitus of crystals growing from their solutions has great potential. Studying peculiarities of human biological fluids (serum of blood, urine, saliva, liquor and so on), they are determined to be unbiased indices of the functional state of an organism. A particular ferroic class, namely ferroelastics (ninhydrin), actively alter its configurations of the forming crystals in the process of crystallization from solutions at comparatively moderate oscillations of super saturation. Depending on conditions there appear whiskers, skeletons, dendrites, staurolites, spherulites. This peculiarity was used at working out crystal morphological method for the purpose of person's disease diagnostics $[1,2,3,4]$.

Varix dilatation is a disease of the whole organism, where homeostasis and microcirculation systems, mineral metabolism, redox processes, enzymatic and hormonal activity get broken. Varix dilatation is polyetiologic disease. Chronic venous insufficiency as one of the actual and complicated problems of present medicine has taken on keen social significance $[4,5,6,7,8]$. However, there is no unified and commonly accepted classification of the disease stages so far. In numerous proposals, authors are guided by an external form, sizes and vein localization $[9,10]$. These classifications are visual, subjective and don't reveal clear differentiation of disease stage. The unified classification urgency does not raise doubts. Its necessity is obvious at solving such questions as terms and volume of medical measures, therapy effectiveness appraisal, at solving questions of work-status and so on.
In connection with the foregoing the most perspective trend in studying venous insufficiency must be a complex analysis, including researches both in direct affection zone and in human systems state on the whole.

The purpose of this paper is identification of varicose disease stages of lower limbs, based on comparative analysis of histological and first applied crystal morphological data of vein structure traits for healthy people and patients with different stages of varix dilatation of lower limbs.

\section{Materials and Methods}

The experiments have been carried out on the biopsies material received during the veinectomy in the surgical clinic of Tver Medical Academy from the patients suffering from varicose disease of different severity degree (80 patients) and from practically healthy people having suffered from lower limbs trauma (8 patients).

For crystal-morphological analysis eliminated areas of veins weighing $0.5-2.0 \mathrm{~g}$ were comminuted using the lancet, then were grated with dry fluvial sand, were mixed with a solution ninhydrin (96\% alcohol) (Sigma, USA), after samples were filtered with filter papers "CHMLAB GROUP 2005". The filtrates were crystallized in the laboratory at $+20^{\circ} \mathrm{C}$ and resulting samples were examined and photographed at the magnifications of $\times 80$ use of the a optical microscope "Biomed-2" № 4F 8663200/39 with camera Nikon D3100 kit AF-SDX Nikkor 18-55mm.

For own standards processing crystal-morphological pictures of pure ninhydrin spirit solution and ninhydrin mixture with homogenates of the vein areas of healthy people and the patients with the first-, second- and third stages of varicose disease of lower limbs have been compared.

\section{Results}


The crystallized pure ninhydrin spirit solution showed the crystals that have the form of spherulites with thin rays (1) diverging from the centre of crystaillization (2) over the radius (Figure 1).

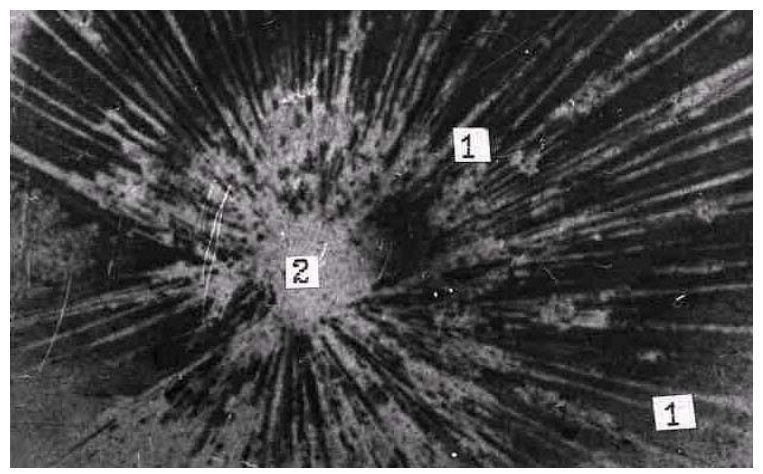

Figure 1. Crystallized solution ninhydrin (96\% alcohol): 1-thin ray; 2-centre of crystallization

Vein wall investigations conducted and literary data [5] permitted to determine veins taking an active part in hemodynamics. Before the beginning of forming unbiased factors of varicose disease vessels walls undergo certain changes, worsening their feeding and weakening venous tonicity.

Microscopic vein wall indices of the test group were within the norm. All three vein walls were distinctly conveyed, demarcated and had the same thickness along the full length (Figure 2). The internal layer - intima (1), is presented by endothelial, unstriated and connectivetissue fibers. The medial layer - media (2), is formed by 8-10 rows of muscular strata, surrounded by collagenous and elastic fibers. The external, adventitious layer (3) consisted of collagenous and elastic fibers, a small amount of longitudinally located unstriated fibers and spongiose bindweb.

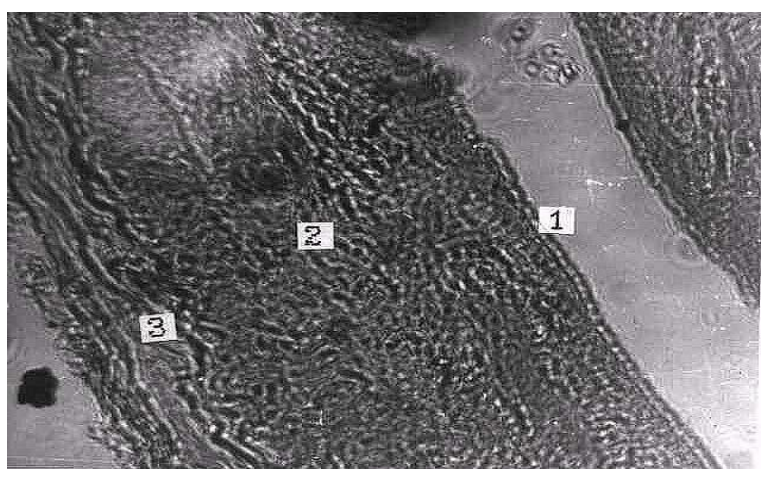

Figure 2. Microscopic indices of vein wall of the test group: 1-internal layer of vein - intima; 2-medial layer of vein endothelial layers of cells; 3-external, adventitious layer of vein

The alteration of chemical composition of human organism tissues and liquids is known to be the result of pathological process, it can be fixed crystallizing the body of interest. The habitus change, concerning the norm can be used as a diagnostic factor [11].

Crystal-morphological researches of the crystallized vein areas of healthy people with ninhydrin spirit solution showed the crystals to have the form of semispherulites, acerate rays diverge from the centre of crystallization over the radius (1). They are thin, have the same length (Figure 3).

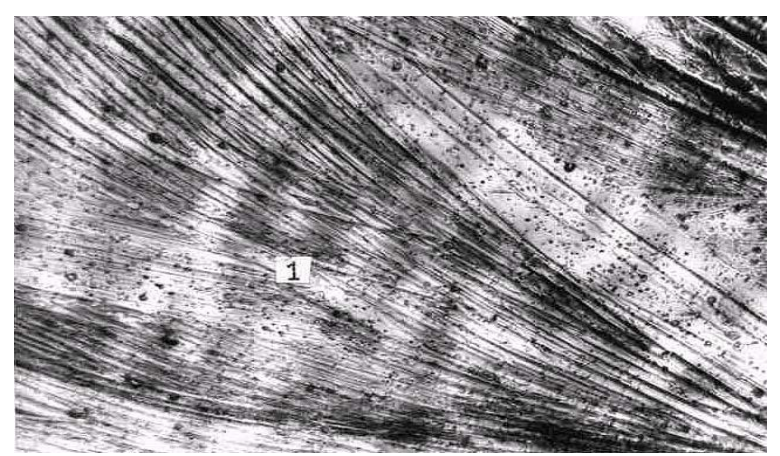

Figure 3. Crystallized vein areas of healthy people with ninhydrin spirit solution: 1-rays diverging from the centre of crystallization

Vein walls investigations of patients suffering from the first stage varicose disease showed that all three layers of the vein wall were subjected to some change. There appears their thickening owing to hypertrophy of the forming cells, which leads to the vein wall node (Figure 4) and results in its lumen narrowing, blood transport problem.

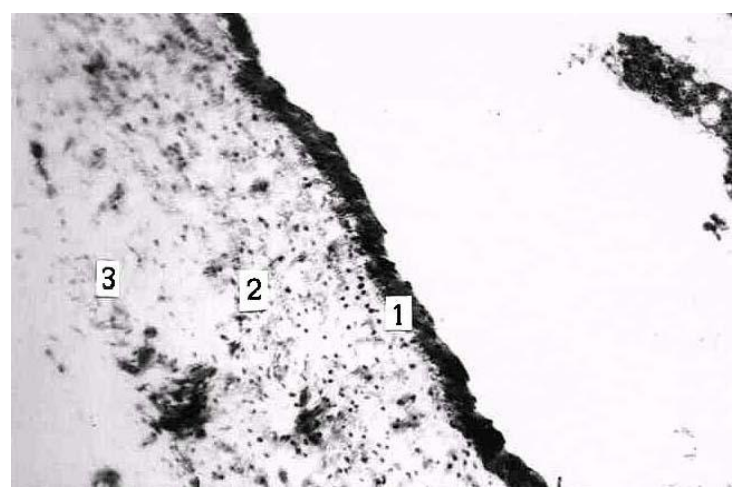

Figure 4. Vein wall of the patients suffering from the first stage varicose disease: 1-endothelial layers of cells; 2-media; 3-adventitious layer of vein

The process begins with changing structure of endothelial layers of cells (1), which retain a continuous line but obtain an edematous appearance that points to their functional activity increase.

At the first stage of the disease the crystals gain the form of spherulites. The first order branches go aside from the basal trunk (1), then interacting with the adjacent crystals complex branching starts (2). Formally one can count not only the second but the third order branches (Figure 5).

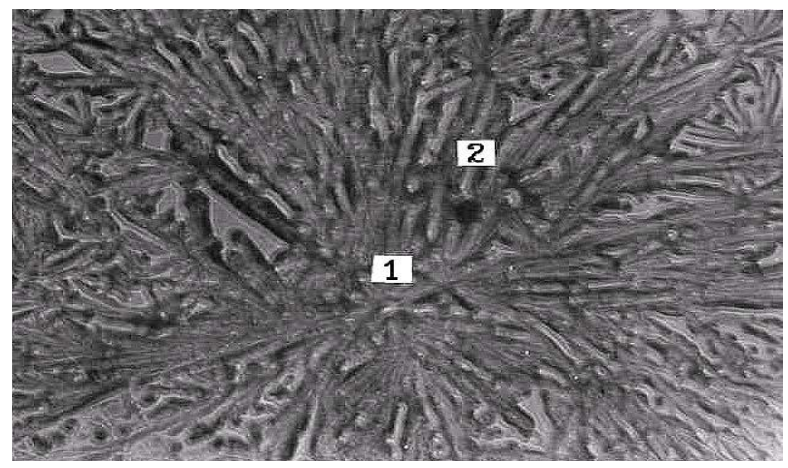

Figure 5. Crystallized vein areas of the patients with the first stage varicose disease with ninhydrin spirit solution: 1-basal trunk of spherulites; 2-complex branching rays 
This effect is the result of the crossed branches contacts of the neighboring dendrites which look fused. Under microscopic investigation chemical composition changes of the vein cells are revealed as cell hypertrophy and metabolism process increase, change substrate, it becomes viscous, and in turn it puts crystals into a special shape.

Thus the alternation of semispherulites with spherulites is marked in kinetics of crystal growth which is connected with absconded physico-chemical processes taking place in the vein walls. At that the first stage of varicose disease is not clinically exhibited [12,13,14].

At the second stage of varicose disease microscopic investigations allowed to reveal a whole series of alterations, concerning control, testifying to pathological process in vascular wall. There is no distinct demarcation of layers, they lose an elastic framework, have an irregular thickness along the whole length. Endothelial cells are partly destroyed (1), and plasmalemma integrity of others is here and there disturbed, it has lesser extension due to the leveling of folds. The lysis of plasmalemma is accompanied by cytoplasm going out into the opening of the vessel (Figure 6).

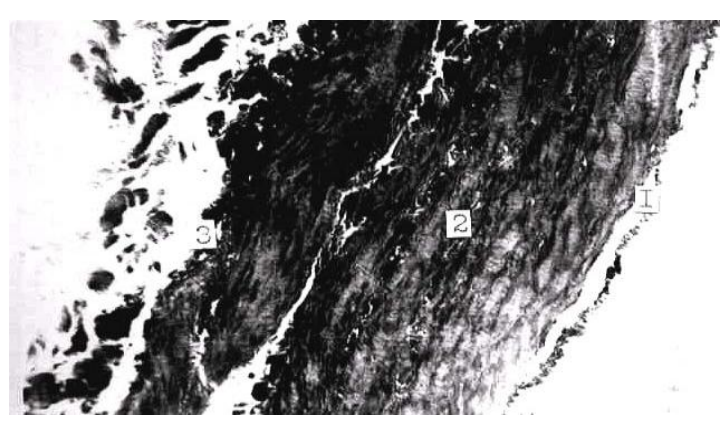

Figure 6. Vein wall of the patients suffering from the second stage varicose disease: 1-partly destroyed endothelial cells of vein wall; 2-media; 3-adventitious layer of vein

According to the literature such alterations are the starting moment of thromb forming [5,7,9].

Examining vein wall structure of the patients with the second stage varicose disease the process of unstriated muscular tissue degeneration into adipose one is marked. At vein crystallization of the patients with the second stage varicose disease the crystals grow in the general form of semispherulites. However the rays are bent in spicate way, issue in six-seven from the crystallization centre (1), form additional second order branches $(2,3)$ (Figure 7). The rays have a complicated structure of lateral faces, in the form of thin, transparent plates located at the angle of 30 degrees.

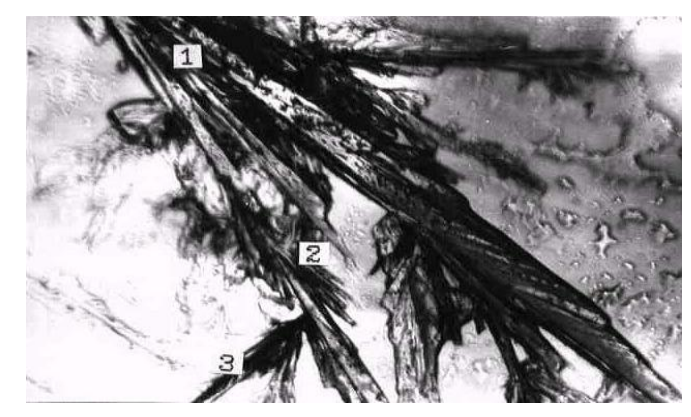

Figure 7. Crystallized vein areas of the patients with the second stage varicose disease with ninhydrin spirit solution: 1 - the centre of crystallization; 2 - branches of secondary order of semispherulites; 3 - additional second order branches of semispherulites
Such structure is explained by the peculiarity of adipose tissue crystallization prevailing in the vein wall in connection with replacing unstriated fibers with adipose cells.

In particular nascent as affected by adipose tissue dendrite crystallization with complicated structure of crystal ray edges is the consequence of impurities availability, which accumulates near the edges and blocks their growth.

The third stage of varicose disease is characterized by irreversible, degenerative processes. The demarcation of layers and elastic framework between them are completely absent. The layers have a different thickness along the whole length of vein. As a whole the wall gets thinner. Through the light microscope intima is not determined (1), endothelial cells are destroyed along the whole length of vein, allowing to suppose sharp decrease of protective and nutrient function of the vein wall (Figure 8).

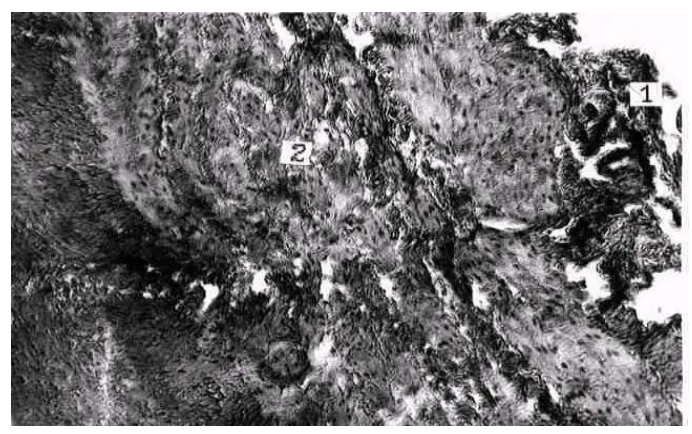

Figure 8. Vein wall of the patients suffering from the third stage varicose disease: 1-destroyed intima; 2-media

Significant functions belong to the endothelial layer: it forms a vessel lining, delimits blood from the environment; contributes to substance transport both towards the wall from blood and backwards. Hence it fulfils a barrier and nutrient function and is the first to react to the developing pathological processes.

However similar changes in the vein wall can cause not only varix dilatation but other diseases, or age-related changes [7].

So crystalomorphological method is offered for more precise differentiation of varicose disease stages.

At the third stage of varicose disease crystalforming body of interest contains an abundance of erythrocytes (considerable capillaries neoplasm contributes to). As it was clarified during the experiments on the whole blood crystallization, erythrocytes are not the areas of the crystal growth centres, they stick together and are collected in groups (2). So at the third stage the crystals are not formed or grow as single, big (1), incrassate rays (Figure 9).

The emergence of atypical forms points to drastic homeostasis disorder. At the third stage of varicose disease when irreversible degenerative processes clinically and morphologically develop in the vein wall, crystalomorphological method confirms it by visual demonstration.

\section{Discussion}

The process of crystal forming is known to be sensitive to the presence of impurities. This viewpoint on the crystal growth is dominant in modern crystallography. 


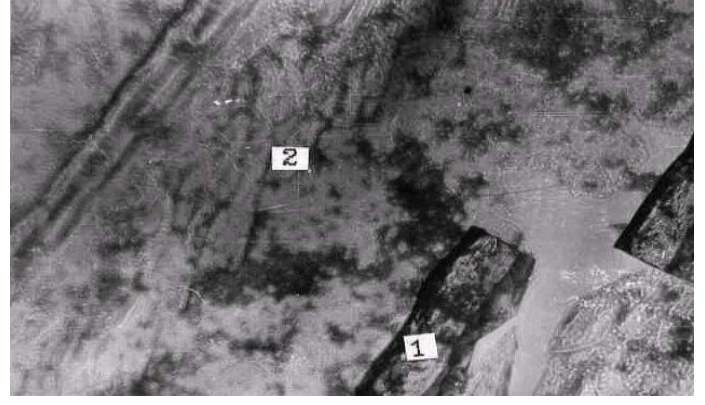

Figure 9. Crystallized vein areas of the patients with the third stage varicose disease with ninhydrin spirit solution: 1-single, big crystals; 2-stuck together erythrocytes in groups

Chemical composition change of the vein wall influences the crystal appearance. Therefore the fact of revealing characteristic regularities of the crystal form change at different stages of varicose disease is of great interest.

The crystalomorphological method was applied in classification of varicose veins stages. In the first stage of disease crystallograms of vein walls with ninhydrin had the form of spherulites, in the second stage - the form of whiskers, in the third one the crystals did not grow $[13,14,15]$. The results were compared with diagnosis. Later on the more precise definition of methods would allow to trace the process of treatment, recovery of the afflicted people and the regularity of changing crystal forms according to the changes of chemical composition of biological mediums under conditions of various pathology.

Thus when studying crystalomorphological pictures of the vein walls of healthy people and the patients with varicose disease of lower limbs cellular composition changes of blood vessels walls and differentiations of textures are marked depending on stages of varicose disease.

\section{Conclusions}

These researches give grounds to consider that crystalomorphological method of the stage appraisal of varicose disease of lower limbs (in comparison with notorious and added to armoury in surgery) is more informative, representative and definite. It permits to judge of the degree, sequence, regularity and character of venous pathology at every stage of disease. It affords a basis to recommend it to applied health service.

\section{Acknowledgement}

This work was performed within Department of Biology, Tver State Medical Academy and Department of Crystallization, Tver State University.

Permission to use the equipment and support of the Sharing Service Center of Tver State University is greatly acknowledged.

\section{References}

[1] Gordienko A.N., Tarabanchuk V.P., Kurbatova L.A., Smirnov Y.M., "Crystalomorphology of Liquor Systems - Diagnostic Substance,” Physics of Crystallization, 97-100. 1988.

[2] Komarov I.A., Khomullo G.V., Smirnov Y.M., Kurbatova L.A. "Crystalomorphological Characteristics of Veins at Varicose Disease," Physics of Crystallization, 17-21. 1994.

[3] Kurbatova L.A., Smirnov Y.M., "Crystalomorphological Method Application in Surgery" in Science conference materials, TGMI, 57.

[4] Kozhinova L.A., Maslennikova L.S., "Of Using Crystalomorphological Methods of Analysis in Medicine," Laborotornoye delo, 12. 783-740. 1988.

[5] Vedensky A.N, Varicose Disease, Meditsina, Moscow, 1987, 24.

[6] Zhdanov D.A., Shakhlamov V.A., "Comparative Electronicmicroscopic Investigation of Blood and Lymphatic Capillar Structure," Arkh. Anat, 47. 113-187. 1964.

[7] Zenin A.A., Pathological Physiology, Meditsina, Moscow, 429. 1992 ,

[8] Menshikov V.V., "Laboratory Research Methods in Clinic," Meditsina, 174-177, 1987.

[9] Makeeva G.F., To the Question of Surgical Methods of Varix Dilatation of Lower Limbs Therapy and Their Afterhistory, Thesis of Candidate of medical science, Moniki, Moscow, 44-46. 1989.

[10] Kurbatova L.A., Philipov A.N., Smirnov Y.M., Gordienko A.N., Voronkova L.N., Diagnostic method of central nervous system diseases, Inventors certificate 1412738, USSR, Moscow, 1-2. 1985 .

[11] Kalikshtein D.B., Moroz L.A., Kvitko N.N., "Crystalographical Researches of Biological Substrates", Clinical medicine, 68 (47). 28-31. 1990.

[12] Kurbatova L.A., "Diagnostic Features of Crystallization in Biological Environment" in The Thirteenth International Conference on Crystal Growth in Conjunction with The Eleventh International Conference on Vapor Growth and Epitaxy Doshisha University Kyoto, Japan, 04p- S14-12.

[13] Kurbatova L.A., Haritonova E.A., Shestakova V.G. "Crystallomorphological method of des of the varicose disease of lower extremitiesiagnosti," Natural and humanism, 2006.

[14] Kurbatova L.A., Smirnov I.M., "Identification of the nervous system diseases by crystal-morphological method" in Collected materials Troitskaya V Conference "Medical Physics and Innovations in Medicine", Trovant, 2, 307-309.

[15] Kurbatova L.A., Crystallization in biological environment and its application in the medicine, Abstract of the thesis on competition of the academic degree of the candidate of the chemical sciences, TvGU, Tver, 1-20. 1995. 